\title{
Conformance Testing of a Multimedia System Using PHACT
}

\section{L.M.G. Feijs.}

Philips Research Laboratories Eindhoven,

Eindhoven University of Technology.

EUT, P.O. Box 513, 5600 MB Eindhoven, The Netherlands.

Tel.+31 402742953, Email:feijs@natlab.research.philips.com

F.A.C. Meijs, J.R. Moonen.

Philips Research Laboratories Eindhoven.

PRLE, Prof. Holstlaan 4, 5656 AA Eindhoven, The Netherlands.

Tel.+31 402743932, Email: \{meijs, jmoonen\}@natlab.research.philips.com

\section{J.J. van Wamel.}

Centre for Mathematics and Computer Science. CWI, P.O. Box 94079, 1090 GB Amsterdam, The Netherlands.

Tel. +31 205924099, Email: Jos.van.Wamel@cwi.nl

\begin{abstract}
In this paper we report on the applicability of a number of ISO-9646 techniques, concepts and automatic test-generation tools in the area of multimedia processing (MPEG2). Several concepts, such as PICS and PCO, and EFSM-based TTCN generation carried over to this new domain very well. The PHACT tool environment, originally developed at Philips for hardware (VHDL) protocol testing, was adapted and used for test generation and test execution in the multimedia processing domain. The paper also highlights a number of interesting issues related to the usage of (E)FSMs, such as dealing with reactive behavior at a software API PCO and dealing with stream-PCOs. Multimedia streams were modeled as FSMs. This turned out to be a new application area for test generation from composite or product FSMs, a technique originating from the embedded testing method. The process followed for test preparation and test execution consists of seven phases, which are surveyed in the paper.
\end{abstract}

Keywords

Test Generation, PICS, EFSM, TTCN, MSC, Conformance Testing, Multimedia Testing, MPEG, DVB, Digital Television 


\section{INTRODUCTION}

It is widely recognised that more and more $\mathrm{CE}$ (consumer electronics) products are becoming part of distributed, open systems. From the area of telecommunication systems, we known that for interoperability of the constituting parts of a system, it is essential that these parts are tested on functional conformance with respect to internationally agreed standards. This has led to the ISO9646 methodology [12], the language TTCN [13], and a wealth of techniques for automatic test generation, notably (E)FSM-based test-generation $[1,10,17,20]$. Thus it seemed promising to apply these concepts and techniques to the CE domain, which used to be based on analog techniques, but which is progressing into digital coding and transmission techniques. Furthermore, we consider functional conformance testing to be as important for hardware as it is for software.

At Philips a one-chip DVB (Digital Video Broadcast) source decoder is being developed. DVB systems are digital television systems based on MPEG2-compressed audio and video, which have inherent technical advantages over conventional systems [2]. The core of the Philips DVB decoder system is the DIVA5 IC, which integrates all functionality required for receiving and decoding MPEG2 transport streams, including descrambling, demultiplexing, audio and video decompression, overlay graphics provisions, and analog television encoding. It also contains an embedded microprocessor and several peripheral interfaces. The DIVA5 is therefore capable of performing all controller tasks in digital television applications such as set-top boxes.

Conformance testing usually concerns system characteristics which are of interest for interconnectivity with other systems. In the DIVA5 project, this means that we are interested in whether the system correctly processes MPEG2-compliant bit streams, as specified by the international standards for MPEG2 [11] and DVB $[7,8,9]$.

We started from a test tool environment for protocol conformance testing of hardware designs in VHDL called PHACT (PHilips Automated Conformance Tester), which was developed during 1995 and 1996, as reported in IWTCS'97 [18]. PHACT was applied to the development of both the DIVA5 hardware and software. During the project the test environment was adapted to obtain an environment specifically suited for DIVA5 compliance testing, while the generic overall structure of the tool environment was maintained.

The main experiences we would like to report in the present paper are the following. Firstly, we found several ISO-9646 concepts such as PICS and PCO applicable in the multimedia processing area. Secondly, we found EFSM-based test generation very useful, but it had to be complemented with other techniques, notably for data-aspects (stream construction). Next to that, the paper highlights a number of interesting issues related to the usage of (E)FSMs, such as dealing with reactive behavior at a software API PCO (Point of Control and Observation), and dealing with a stream-PCO. Modeling multimedia streams as FSMs also turned out to be a new application area for test generation from composite or product EFSMs, a technique originating from the embedded testing method. 
Section 2 surveys the followed test preparation and execution process. Section 3 discusses test preparation in more detail. In Section 4 the various types of tests needed for MPEG conformance testing are treated. Section 5 is about the test tools (PHACT) and the way they were used. In Section 6 the application of composite EFSMs is discussed. Section 7 contains a general discussion on test coverage. Finally, Section 8 contains some concluding remarks.

\section{SURVEY OF THE TEST PROCESS}

Although there are systematic test sets for video decoders (e.g., the Sarnoff stress tests [19], as sold by Doctor Design Inc.), we were, and are, not aware of any commercially available, systematic test sets that cover the MPEG2 systems layer. Furthermore, our job was not only to test the MPEG2 systems stream processing in isolation, but also the various programmable control registers and software of the DIVA5 chip. This meant that we had to start from scratch. The approach is schematically shown in Figure 1.

In the conformance testing of communication protocols, Protocol Implementation Conformance Statement (PICS) proformas are used, in order to make statements about which capabilities and options of a protocol have been implemented by a product supplier $[14,16]$. A completed PICS proforma can therefore be used as a basis for the definition of tests for a product. In an analogous way, we formulated a PICS proforma for DVB source decoders such as the DIVA5 system. In that document, system requirements were listed which were derived from the international standards for MPEG2 and DVB.

The following step was to describe a large number of test purposes, which are informal descriptions of tests, in such a way that the PICS items are adequately covered. In the formulation of test purposes we also used the DIVA5 systems specification, in order to provide sufficient detail for the implementation of the actual tests. Implementation details often turned out to generate specific test purposes.

The next step was the definition of the actual tests that were run on the implementation by filling out so called test templates. Each completed test template aims to cover a number of individual test purposes, in order to keep the total number of executed tests manageable.

A test template may contain an Extended Finite State Machine (EFSM) for modeling interactive aspects of the DIVA5 system, as it takes place at the software Application Programmers Interface (API) or hardware/software interface. These EFSMs were used as a basis for automatic test generation in the TTCN (Tree and Tabular Combined Notation) formalism [13]. In the test construction phase, the EFSMs were implemented, TTCN tests were generated and subsequently connected to the DIVA5 by additional software, which we will subsequently call mapping software. TTCN tests were executed by our test environment PHACT.

The results of EFSM-based tests are visualised using Message Sequence Charts (MSCs) [4]. For any test, the result is summarised by a verdict, which is either pass 


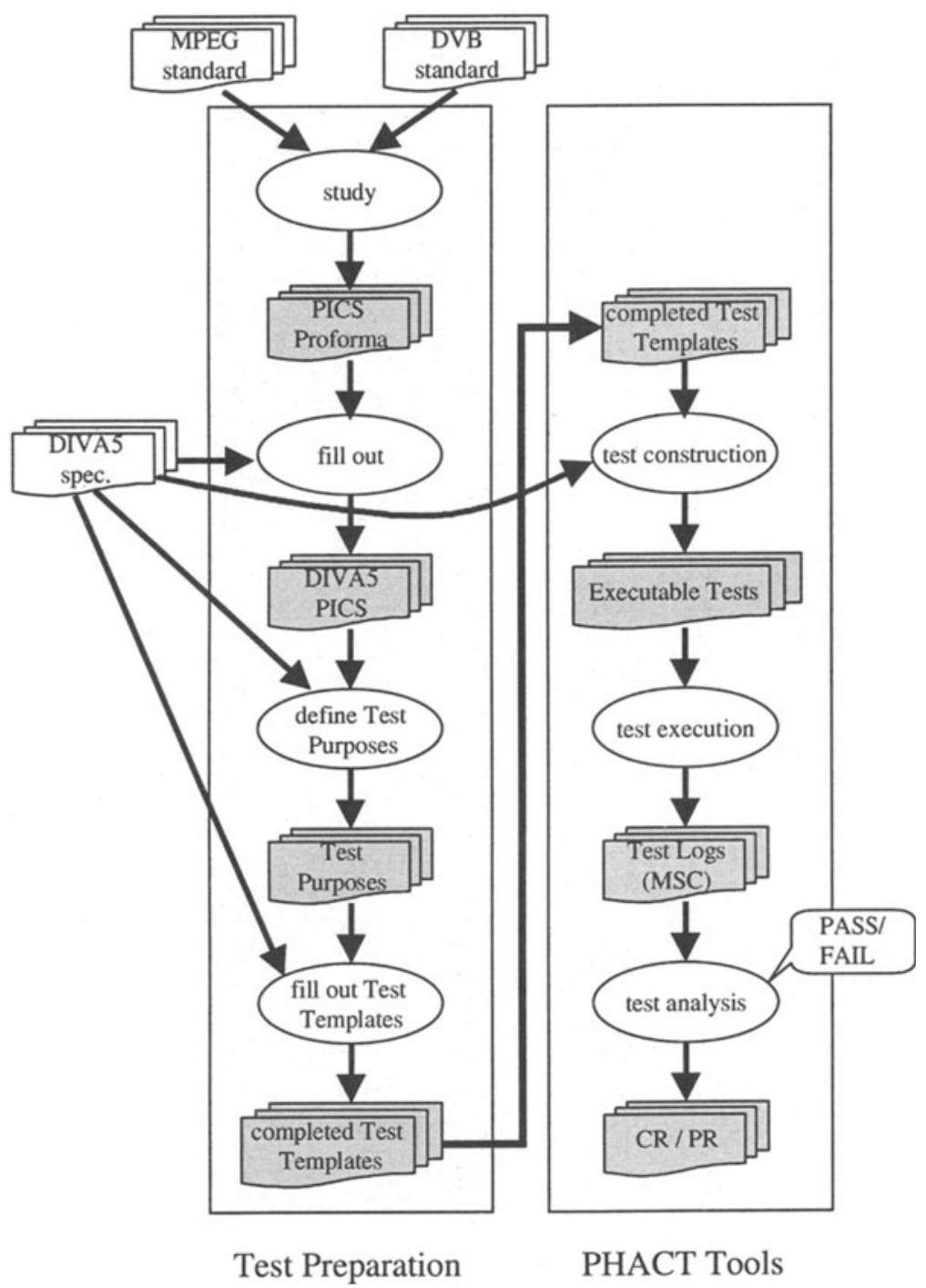

Figure 1 Survey of the Test Process. 
or fail. 'Fails' generally lead to Change Requests (CRs) or Problem Reports (PRs) for the implementation under test.

\section{TEST PREPARATION}

In this section we describe the necessary steps that precede the actual testing. The test preparation process consists of three phases:

1. design and filling out of a PICS proforma;

2. identification of test purposes;

3. filling out of test templates.

We discuss each phase now, starting with the PICS proforma, which we had to design for MPEG2/DVB decoders. The proforma contains requirements from three categories:

1. MPEG2 decoder characteristics (hardware claims),

2. DVB specific features (application software claims),

3. DIVA5 API functionality (system software claims),

based on the ISO 'Systems' and 'Compliance' MPEG-2 standards [11], the ETSI DVB standards, and the Philips DIVA5 API specification, respectively. In Table 1, some PICS items are given as an example.

\begin{tabular}{|c|c|c|c|c|}
\hline ITEM & REQUIREMENT & STAT. & REF. & SUPP. \\
\hline A.1.13.a & $\begin{array}{l}\text { For error conditions that do not result in un- } \\
\text { acceptable decoding artefacts, does the system } \\
\text { maintain the system time base in the event of } \\
\text { errored or missing packets that carry PCRs? }\end{array}$ & $\mathbf{M}$ & {$[11]: 8.1 .8 .2$} & $\mathrm{Y} / \mathrm{N}$ \\
\hline A.5.2 & $\begin{array}{l}\text { transport_error_indicator: If this bit is } \\
\text { set, does the system invoke a suitable con- } \\
\text { cealment or error recovery mechanism? }\end{array}$ & 0 & {$[7]: 5.4$} & $\mathrm{Y} / \mathrm{N}$ \\
\hline A.10.4 & $\begin{array}{l}\text { Does the system operate over the full range } \\
\text { of the system clock frequency specified in } \\
{[11]: 2.4 .2 .1(27 \mathrm{MHz} . \pm 810 \mathrm{~Hz} .) ?}\end{array}$ & $\mathbf{M}$ & {$[7]: 5.3$} & $\mathrm{Y} / \mathrm{N}$ \\
\hline
\end{tabular}

Table 1 Examples of PICS items 
Next we discuss the test purposes, which follow the PICS as a kind of step-wise refinement. A PICS may contain a large number of hardware and software claims, but it states in no way how such claims can be verified. In order to fill this gap, the following step was to informally describe a set of tests, such that all relevant PICS items were adequately covered. Since it now came to relate PICS items to hardware and implementation issues, the DIVA5 specification had to be scrutinised.

The informal test definitions were called test purposes. Test purposes state what DIVA5 functions have to be tested, and possibly how. The test purposes are related to specific system components and control registers of the DIVA5, to the relevant parts of the software API, and to specific PICS items, or conformance requirements.

The test purposes are grouped into so-called test groups. Each test purpose was described as a record, using keywords, as in the following example taken from Test Suite 11: Handling of duplicate, errored, and missing transport stream packets.

$\begin{array}{ll}\text { Test purpose } 11.5 & \text { : Section filter response to duplicate packets. } \\ \text { Test method } & \text { : Inspection of buffer contents. } \\ \text { Streams } & \text { : DPS with pairs of duplicate (P)SI packets. } \\ \text { DIVA5 component } & \text { : PID filter (CC), section filter. } \\ \text { Pics items } & \text { : A.5.5.a. } \\ \text { Project stages } & \text { : VHDL. }\end{array}$

A DPS is a Dedicated Packet Sequence, a short stream that is constructed for testing some specific functional behaviour of the system, with no real-time audio or video contents. Please note that EFSM-based testing is only one of the test methods, next to auditory/visual inspection and inspection of control registers or memory contents. Other keywords were used as well, including Interrupts, $R, W, R / W$-Register(s) (Read, Write, Read/Write control registers). Next to the VHDL stage, there are test purposes best covered in the QuickTurn phase (QT), where a hardware emulator, the QuickTurn, is used, or in the IC phase where the real silicon is used.

Finally we discuss the last step of the test preparation phase, the completion of so called test templates. These are documentation forms, used to describe a single test. For practical reasons we limited the total number of test templates and test streams by allowing a single test template to cover a number of individual test purposes. The test template form reflects the fact that a typical test is a pair $\langle s, t\rangle$, where $s$ is a stream, for example a DPS, and where $t$ is a test script, for example an EFSM together with additional mapping software to translate concrete events and data-structures into the abstract events of the EFSM, and vice versa. For cross-referencing purposes, the templates have an entry where the test purposes that are covered by the test are included. 


\section{TYPES OF TESTS}

Before presenting the tools proper, we must discuss the distinct types of tests. To execute a test on the DIVA5, both an MPEG2 test stream and a test script are necessary. The stream carries MPEG audio, video and data, and is played in the test execution environment by a bit-stream player (BSP). During testing, the BSP plays the role of an MPEG/DVB broadcaster. The test script controls the settings of various parameters of the DIVA5 system, specific for a test. It plays the role of a set-top box application on top of the software API of DIVA5.

A distinction can be made between tests where the focus is on the 'stream' part, and tests where the focus is on the 'script' part. In general, a test script consists of a prescribed interaction pattern: a list of (stimulus, reaction) tuples. Execution of such a script basically boils down to the application of the stimuli to the system and observation of the system's reactions. When the interaction pattern is as prescribed, the test verdict will be pass, otherwise it will be fail. In our case, test scripts take the form of a TTCN test suite generated from an EFSM specification of the system.

Tests where the focus is on the script part typically test the system's programmable control registers and interrupts. While a stream is playing, the control registers are reprogrammed and the system's reaction is observed either via interrupts or via status registers. The reprogramming as well as the monitoring is controlled by the test script.

Since we aim at automatic test execution and analysis, the system's reaction should be detectable by our test software. For some types of tests, however, this is not a feasible or the most convenient method. For example, in order to determine the correct presentation of audio and video in the presence of time-base discontinuities, we also constructed tests that have to be verified by simply observing the audio and video as the system presents it. For such tests, the test script degenerates to an initial configuration of the system, and test verdicts will have to be issued by the human observer. These tests are called stream tests, and EFSM-based test script generation will not be used here.

Another type of stream tests are tests where there is no direct, observable reaction to a stimulus supplied to the system. For example, for testing whether some filter extracts the right data from a stream, it is more convenient to simply program the filter, play the stream, route the output of the filter to memory and inspect the memory contents afterwards.

For script tests, we could often abstract from parts of the contents of the stream; the packet payload. For example, for a test of the 'MPEG-Systems' buffer management only the size of the buffered data matters, not the specific contents. This does not imply that the stream may be constructed completely independent of the test script. For example, when the control registers of the descrambler module are tested, the stream must contain scrambled data at appropriate positions in the stream. But, in general, streams for tests focussed on scripts tend to be less complex than for stream tests. 


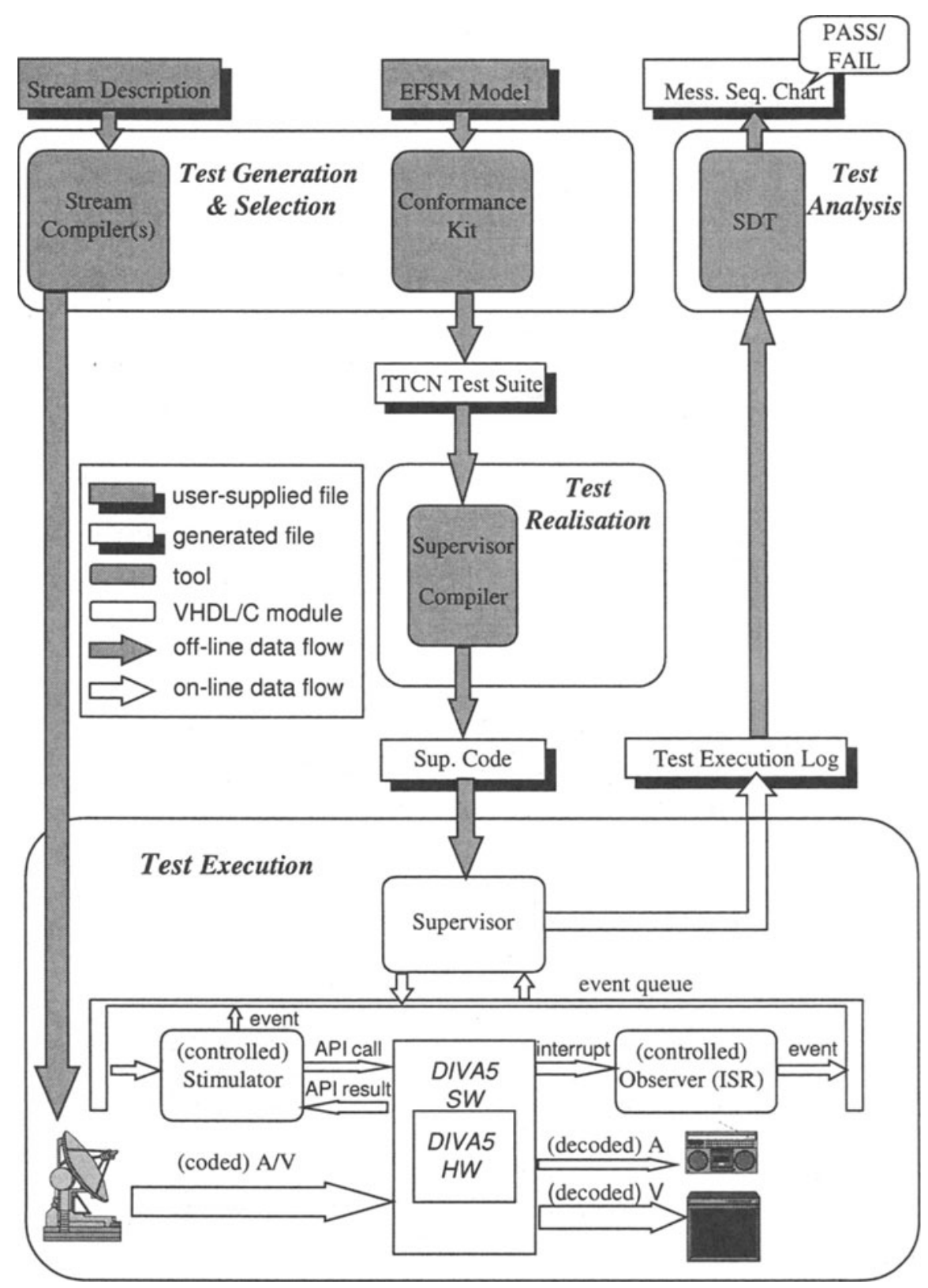

Figure 2 The PHACT test tool environment

\section{TOOLS}

Since both a stream and a test script are necessary to execute at test, the tools used in test development therefore belong to two different categories: (1) stream generation 
tools, (2) test script generation and execution tools. The first category is needed for the construction and validation of MPEG2-compliant bit streams, the second category serves for control of the test execution.

PHACT, as it has evolved now, is shown in Figure 2. The design of PHACT has been reported on in [18], hence we will not explain all details here. We only mention that the Conformance Kit [3] is used to generate a set of tests in TTCN format from an EFSM specification. An example TTCN test case is given below.

\section{Test Case Dynamic Behaviour}

\begin{tabular}{ll}
\hline Test Case Name: & test_2 \\
Test Group: & buffers3/pt/filling_odd/ \\
Purpose: & test input "allocate_even_buffer" in state "filling_odd" \\
Defaults Reference: & general_default \\
\hline
\end{tabular}

\begin{tabular}{|c|c|c|c|}
\hline $\mathbf{N r}$ & Behaviour Description & Constr. Ref. & Verdict \\
\hline 1 & + ts_filling_odd & & \\
\hline 2 & System ! allocate_even_buffer & & \\
\hline 3 & System ? odd_buffer_full & & \\
\hline 4 & System ! allocate_odd_buffer & & \\
\hline 5 & System? even_buffer_full & & PASS \\
\hline
\end{tabular}

\section{Detailed Comments:}

line 1: transfer to state "filling_odd"

line 2: check input "allocate_even_buffer"

line 4: SIOS on "filling_even"

This TTCN is subsequently compiled and combined with mapping information (as explained below) in order to produce an executable test. The test execution environment consists of a stimulator and observer, associated with the software API PCO, a stream-input PCO (coded audio, video and data) and a stream-output PCO (decoded audio and video). The stream-input PCO poses some limitations: it can only be offered an off-line prepared stream. In practice, bit-stream players have no capability to dynamically choose between packets, construct packets on-the-fly, etc. Concerning the stream-output PCO, it should be noted that it is often not feasible to check the output automatically and the decoded stream has to be checked audio-visually (i.e., by a human observer).

We are aware that, due to the Conformance Kit tool, standard formal specifications like SDL are not usable without a translation into the input language of this tool. However, since standard formal specification languages are not commonly used in the CE domain, we did not consider this to be a serious disadvantage.

Tests have been executed on three subsequent platforms: modeled or simulated hardware only, emulated hardware with software and the final hardware product with software. 
We also like to mention that the output of the test execution phase is a test log in a format that can be visualised as a Message Sequence Chart (MSC) by the (commercial) tool SDT [21], see Figure 3. Next to the TTCN events flowing between the tester process and the IUT (Implementation Under Test) process, all interrupts that occur during a test are also shown as arrows from the IUT to the ISR (Interrupt Service Routine) process, even when these interrupts are not directly related to a TTCN input. An ISR is a function which gets executed when a specific interrupt occurs. The time at which the events occurred are also shown. TTCN verdicts are shown as MSC conditions. Using MSCs for test logging, as opposed to the more common use of MSCs for test goal specification, turned to out to be very useful, especially for long test runs. Tools used for MPEG stream construction are outside the scope of the paper.

The rest of this section is devoted to the issue of mapping software. In EFSM specifications the input and output events may be described at a very abstract level. For instance, a complicated filter initialisation may be covered by a single input event setup. Such abstraction is often useful to obtain a manageable set of meaningful tests. But when one wants to use the TTCN test suite defined in terms of these abstract events, extra information has to be added for linking the abstract events to concrete software API calls (or hardware register access), and interrupts of the system under test. We call the software which defines this relation mapping software, and mapping software has to be supplied in VHDL for the VHDL-based execution environment, and in $\mathrm{C}$ for the $\mathrm{C}$-based execution environments (for QT \& IC).

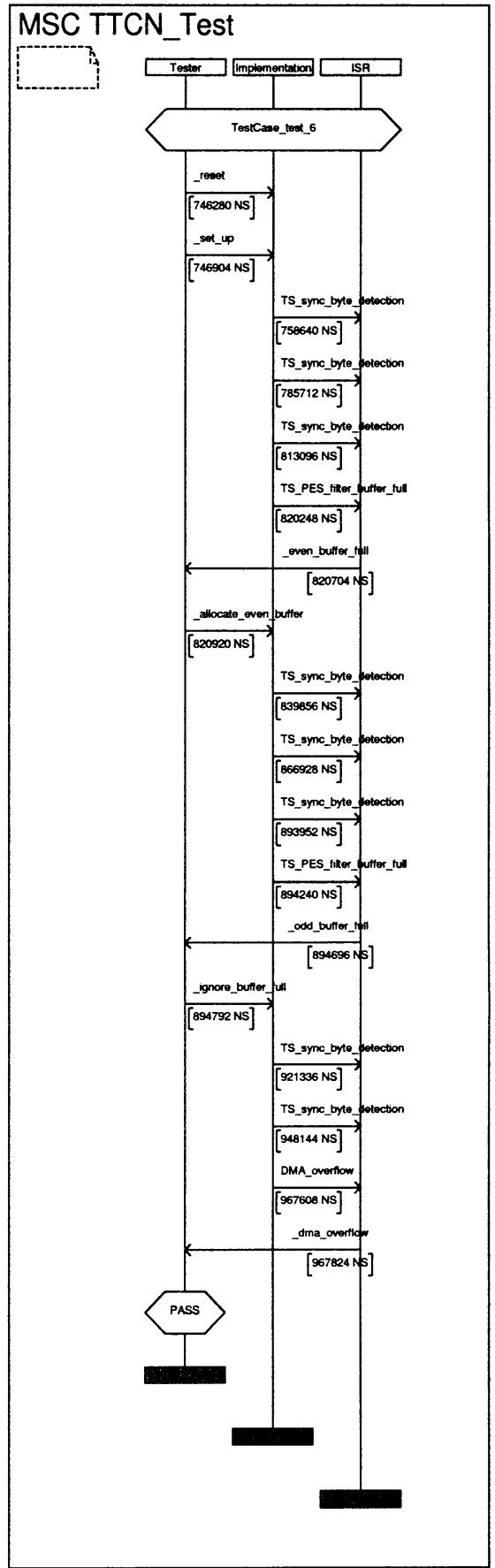

Figure 3 Example Message Sequence Chart 
Basically, mapping software for stimulus events takes the form of case statements which map abstract events to sequences of (lower-level) software API calls. Sometimes, the returned results of these software API calls are the (lower-level) representation of TTCN input events. In such a case, the input event is communicated to the supervisor component (see Figure 2) via the event queue (in Figure 2: the upward pointing arrow leaving the stimulator). For example:

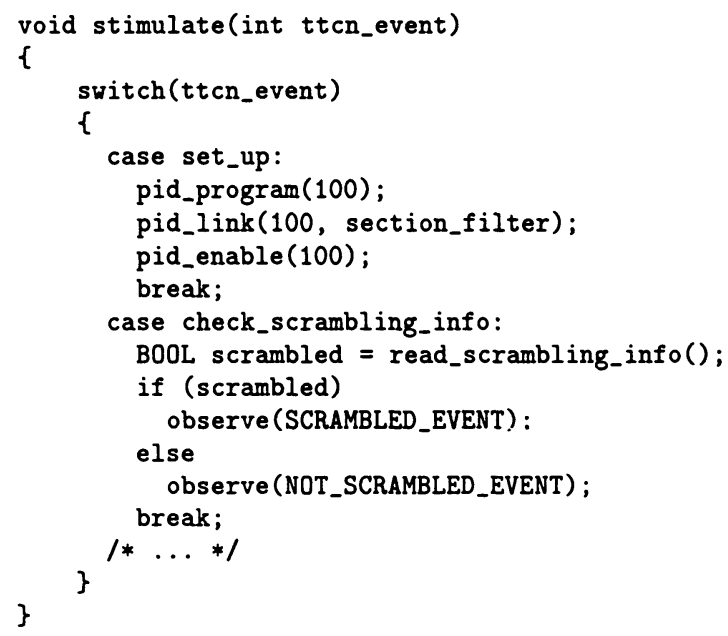

In the above example of stimulus mapping software in a $\mathrm{C}$ environment, both types of stimulus mappings are shown. The first type of stimulus is the most common; a stimulus event is simply mapped to a number of software API calls. The second type of stimulus is actually a read action, where the result of the read is the low level representation of a TTCN input event. The function observe indicates the occurrence of the TTCN input event.

Mapping software for events generated by the system (system output, TTCN input) can also assume the form of a set of interrupt service routines (ISRs), which contain code in their function bodies that indicates the occurrence of a TTCN input event. For example:

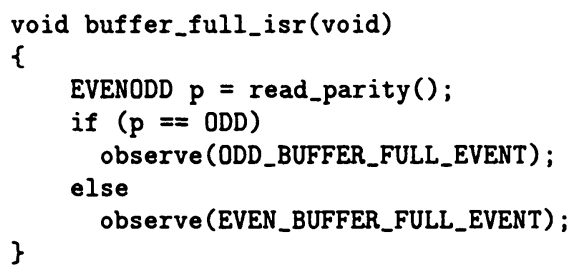

In the above example of observer mapping software in a $\mathrm{C}$ environment, there are two abstract events (ODD/EVEN_BUFFER_FULL_EVENT) related to one concrete interrupt (buffer_full). The function read_parity is used to obtain extra information needed to determine which of the two possible abstract events occurred. This piece of code 
is installed as an interrupt service routine for the buffer_full interrupt before the test execution starts. Most ISRs tend to be one-liners which directly map a physical interrupt to an abstract event, but this does not always have to be the case, as the (real-life) example demonstrates. Figure 4 relates the control and data flow in the above mapping software examples to the test-execution architecture of PHACT.

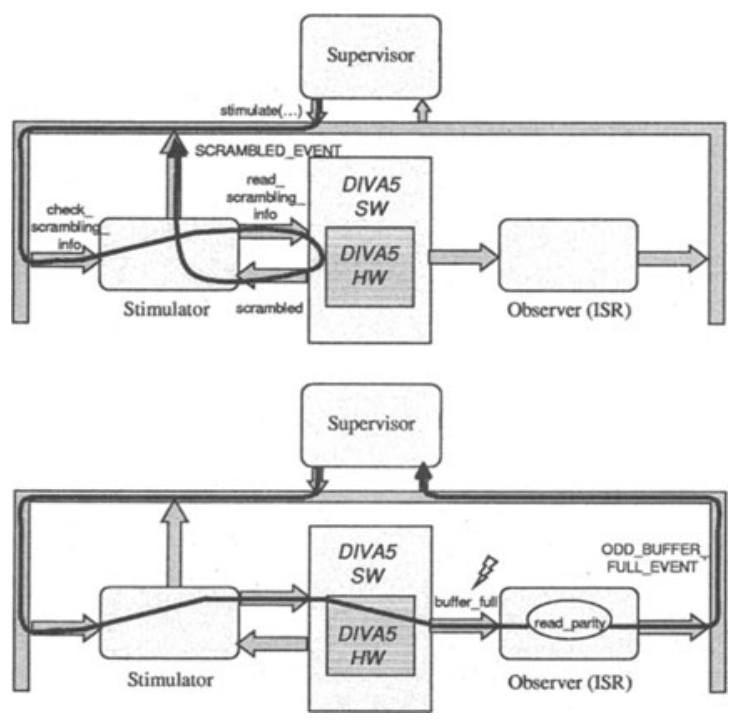

Figure 4 Mapping software control and data flow.

\section{ON THE USE OF COMPOSITE EFSMS}

Usually, there is a close connection between the EFSM used for test script generation and the test stream that is offered to the system during test execution. In such cases, the EFSM becomes a description of some very specific system behaviour, and not general system behaviour description. As a result, many of such specific EFSMs may be needed to achieve a 'good' test coverage (see Section 7). Such EFSMs are also harder to interpret, since they depend on detailed stream information.

In order to make more generic and comprehensible EFSMs we have employed a technique called composite EFSMs. The idea is to specify two (E)FSMs: one EFSM which describes (to a certain extent) a system aspect independent of the stream: the system EFSM, and one FSM which describes a test stream: the stream FSM. 
Now the product or composite EFSM is the EFSM which describes the behaviour of the system when a stream is decoded which conforms to the stream FSM. Symbolically: Composite EFSM = system EFSM $\times$ stream FSM. The Conformance Kit can now generate TTCN from this composite EFSM, where only transitions that originate from the system EFSM - so no transitions from the stream FSM - are taken as test goal* This technique originates from the embedded testing method, and is described in reference [15]. The stream FSM can be considered as test context of the system EFSM.

An increased coverage (compared to the single stream situation) of a specific system aspect can now be obtained by developing a single system EFSM and a number of pairs (stream FSM, stream implementation). TTCN is generated from the composition of the system EFSM and a stream FSM, and each generated TTCN test suite is executed on the system while the corresponding stream is played by a bit stream player in the test execution environment.

We obtain a stream FSM by considering a stream simply as a sequence of packets partitioned or categorised in sequences of a particular packet type. We can then describe a stream by a regular expression in terms of these packet-sequence types. For example, consider a stream that starts with a packet that contains a PCR sample (some kind of MPEG time stamp), which is followed by alternating dummy packets - packets with no relevant payload - and packets with section data. This stream can be described by a regular expression: pcr;(dummy;section)*, and also by the lower FSM of Figure 5.

A system EFSM can be made generic with respect to a set of stream FSMs when no assumptions are made about the order of the various packet-sequences in the stream, but only on the types of packet-sequences in the stream. As a consequence, the system EFSM will have to specify, for all of its states, the reaction upon each 'packet-sequence event' induced by the stream FSM.

A tester now has the option to construct streams that consist of various packet orderings; he only has to update the stream FSMs accordingly. The system EFSM can be used for all streams, without modifications, for EFSM composition and TTCN generation.

For a system EFSM, input events are either connected to the stream FSM ('packetsequence events'), or unconnected, in which case they correspond to software API calls given to the system. All EFSM output events are unconnected to the stream FSM, and correspond to interrupts or software API call results generated by the system.

In Figure 5 an example composite EFSM is shown. The emphasised events are the 'packet-sequence' events. Reserved event spontaneous is used to model 'outputonly' transitions.

*Transitions originating from the stream FSM are internal, technical artefacts introduced for modeling purposes and are therefore not treated as test goals. 


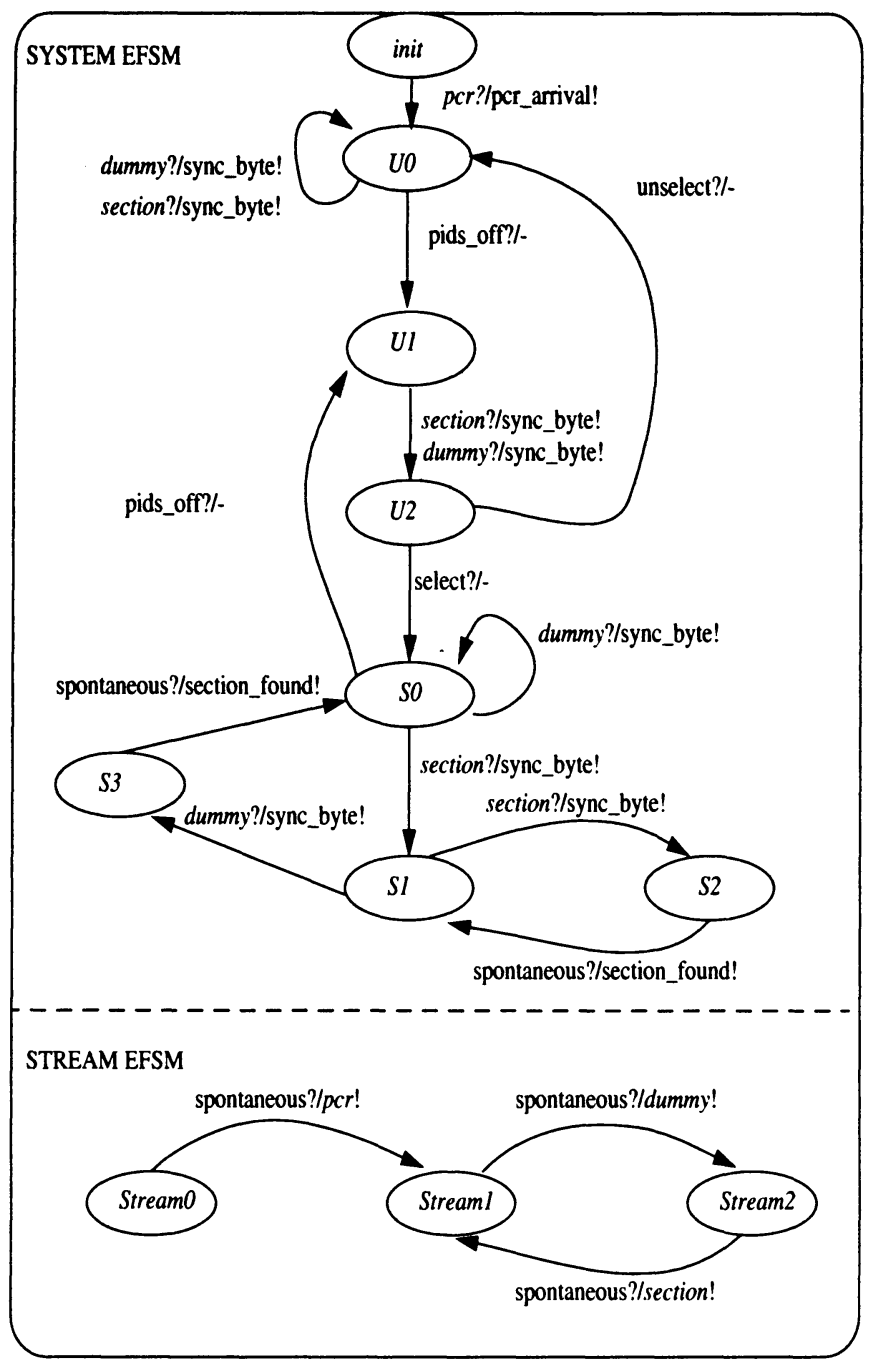

Figure 5 Composite EFSM example

\section{COVERAGE}

This section is devoted to a short discussion on coverage. Although the tests were derived by means of a systematic and step-wise approach, starting from the relevant standards, parts of the hardware specification, and the software API specification, the tests do not give us absolute certainty that the hardware and software are correct and fully compliant. Although some of the considerations may be well-known to the IWTCS audience, we consider it worthwhile to give them here. 
- Firstly, there is the fundamental point put forward by Dijkstra [6], that testing can only reveal the presence of errors, never show their absense. This holds for computer programs, and thus it also holds for the combination of hardware and software.

- Secondly, the specifications of the IUT (implementation under test) are not given as formal models with mathematical rigour. Yes, we were able to cast certain aspects of the system into EFSM behaviour, but these were not all aspects. Moreover, the process of constructing mapping software is a non-exact process in itself.

The first point is much more fundamental than the second, although the present state-of-practice in specification techniques makes both equally relevant. In order to illustrate the first point, we present two arguments.

- Suppose that we would refrain from testing all behaviours of the IUT, but that we would be satisfied to find all things that could go wrong within one second. How many different streams exist which could be fed into the system in one second? With a rate of $40 \mathrm{Mbit} / \mathrm{s}$, there are $2^{40,000,000}$ possible bitsequences that would have to be tried. Even considering only bitsequences conforming to the MPEG syntax, this is a number beyond comprehension.

- Even for those aspects which have been formalised as EFSMs and which have been tested by automatically generated TTCN, let us recall the basis for the completeness claims of this generation process. Even the best variants of the UIO (unique input output sequence)-based test-sequence generation algorithms (like UIOv [23]) only promise to find any defect under the assumption that the faulty implementation (E)FSM does not have more states than the specified (E)FSM. But in practice this assumption need not hold, for example a single integer variable declared and used by the implementor may cause a blow-up of the state space. However, examples where a faulty (E)FSM goes undetected by UIO-based test generation (the test-generated method using in the DIVA5 project) are somewhat academic.

It is also worthwhile to compare (black-box) conformance testing with traditional IC testing (see e.g. the IEEE ITC test conferences [5]). Traditional IC testing is concerned with finding physical defects, e.g. caused by dust particles or wafer anomalies, in ICs which are produced from correct (or assumed correct) designs. When the complexities of the ICs increased in the early 1970s, it was recognised that for finding physical defects, black-box testing was not the way ahead. In the so-called structured testing methods, the details of the design are taken into account. Fault-models are used, based on facts about connection paths which are or are not adjacent. In the DIVA5 project, however, we were testing for (functional) design errors, not for physical defects. Of course, measures have been taken to look into the implementation's source code and to try to find and eliminate errors, but that was not the main task of the test-project. 
In summary, we believe that the obtained test coverage was increased, because of:

1. a systematic test derivation process, compared to traditional, ad-hoc testing, and

2. automation by tool support where possible, compared to purely manual testing.

\section{CONCLUDING REMARKS}

Although MPEG is strictly speaking not an interactive protocol but a data format, the idea of a PICS carried over very well to the multimedia domain. Considering the PICS, it is interesting to note that the MPEG/DVB standards rather define the compliance of streams than the compliance of decoders. Consequently we had to translate stream requirements to decoder requirements.

The intermediate step between PICS and test definition, the definition of test purposes, turned out to be indispensable, since a lot of implementation details are involved in decoding digital TV. We expect that the PICS will become a kind of reusable asset for other conformance testing activities in the area of digital TV.

Applying and adapting our original PHACT tool environment ([18]) to the DIVA5 gave valuable feedback and resulted in two new platforms for PHACT, while maintaining the overall tool structure. Currently PHACT runs on VHDL, pSOS/C (usable for both QT and IC) and UNIX/C environments.

Using MSCs for test logging, as opposed to the more common use of MSCs for test goal specification, turned to out to be very useful, especially for long test runs. Multimedia testing, in particular stream processing, gave rise to a new application area for composite EFSMs, a technique originating from the embedded testing method. Furthermore, one should realise multimedia tests, where the evaluation of test results is simply performed via the evaluation of image and sound quality, do not always allow for simple pass/fail verdicts. In some cases the comparison of various opinions may be necessary, in order to obtain a verdict.

The PICS proforma and the test purposes document led to a large set of tests. The tests themselves revealed various hardware defects and many software and specification/documentation defects. Besides defects directly concerning the test purposes covered by a test, many other defects were found during the process of "getting the test to run". For example, problems were found concerning development tools, bitstream players, peripherals and test-board issues. Finally, it is interesting to note that, although only $40 \%$ of our tests is EFSM-based (the rest was purely stream-based), all hardware errors which were found, we found with these EFSM-based tests.

Future Work. Interesting options for future work in this area are automatic test generation and verdict assignment for stream tests. More research should also go into guidelines for dividing a system into two composite EFSMs. Furthermore, there are other, more recent developments in hardware testing which maybe could be combined fruitfully with our EFSM based work, but we have not investigated this (yet). One such hardware testing technique is 'scan design', which provides that the flipflops in a circuit can be chained into a serial shift-register, called scan chain. Maybe this could be used to inspect an internal state (although this is not black-box, and also, 
reading the scan-chain destroys the system's state). Another hardware testing technique is testability analysis, where propagation of logical values and controllability of variables is analysed in order to select extra test points or partial-scan flip-flops. For a survey of these techniques we refer to [22]. Although we have no experience in ATM testing, we think that parts of our approach could be useful there as well. The MPEG-systems layer addressed in our project, can be compared best with certain variants of AAL, the ATM adaptation layer, notably AAL2.

Acknowledgements. We would like to thank Eric Gijsbers, Joris van den Hurk, Theo Kersjes and Remco Schutte, from Philips Semiconductors, and Ron Koymans, from Philips Research, for initiating and supporting the DIVA5 conformance testing project.

\section{REFERENCES}

[1] A.V. Aho, A.T. Dabhura, D. Lee, M. Umit Uyar. An optimisation technique for protocol conformance test generation based on UIO sequences and rural Chinese postman tours, In: S. Aggerwal, K. Sabnani (Eds.), PSTV VIII (1988).

[2] H. Benoit. Digital television, MPEG-1, MPEG-2 and principles of the DVB system. Arnold, 1997.

[3] S. van de Burgt, J. Kroon, E. Kwast, and H. Wilts. The RNL Conformance Kit, Proceedings 2nd International Workshop on Protocol Test Systems, pp.279-294, North-Holland, 1990.

[4] CCITT. Message Sequence Charts, CCITT Recommendation Z.120, ITUT, 1996.

[5] R. Dekker, F. Beenker, L. Thijssen. Fault Modeling and Test Algorithm Development for Static Random Access Memories, Proceedings Internal Test Conference, IEEE Society Press, pp.343-352, 1988.

[6] E.W. Dijkstra, http://alycia.dementia.org/ strong/public/quotes.

[7] European Broadcasting Union / European Telecommunications Standards Institute. Digital broadcasting systems for television; Implementation guidelines for the use of MPEG2 systems; Video and audio in satellite and cable broadcasting applications. ETR 154 (draft), 1994.

[8] European Broadcasting Union / European Telecommunications Standards Institute. Digital Video Broadcasting (DVB). ETR 211 (final draft), 1996.

[9] European Broadcasting Union / European Telecommunications Standards Institute. Digital broadcasting systems for television, sound and data services; Specification for Service Information (SI) in Digital Video Broadcasting (DVB) systems. ETS 300 468, 1995.

[10] W. Hengeveld, J. Kroon. Using checking sequences for OSI layer conformance testing, In: H. Rudin, C.H. West (Eds.), PSTV VII (1987).

[11] International Organisation for Standardisation. Information technology Generic Coding of Moving Pictures and Associated Audio. - Part 1: 
Systems, Part 2: Video, Part 3: Audio, Part 4: Compliance. International Standard ISO/IEC 13818-\{1,2,3,4\}, 1995.

[12] International Organisation for Standardisation. Information technology Open Systems Interconnection - Conformance Testing Methodology and Framework - Part 1: General concepts. ISO/IEC 9646-1, 1991.

[13] International Organisation for Standardisation. Information technology Open Systems Interconnection - Conformance Testing Methodology and Framework - Part 3: Tree and Tabular Combined Notation. ISO/IEC 9646-3, 1991.

[14] International Organisation for Standardisation. Information technology Open Systems Interconnection - Conformance Testing Methodology and Framework - Part 7: Implementation Conformance Statements. ISO/IEC 9646-7.

[15] H. Kloosterman, H. van Dam, E. Kwast. Test derivation for standardised test methods, In: J. Kroon, R.J. Heijink, E. Brinksma (Eds.), Protocol Test Systems IV (1992).

[16] K.G. Knightson. OSI Protocol Conformance Testing. McGraw-Hill Inc., 1993.

[17] D. Lee, D. Su. Modeling and testing of protocol systems, In M. Kim, S. Kang, and K. Hong, editors, Testing of Communicating Systems, volume 10, pp.339-364. Chapman and Hall, 1997.

[18] J.R. Moonen, J.M.T. Romijn, O. Sies, J.G. Springintveld, L.M.G. Feijs, and R.L.C. Koymans. A two-level approach to automated conformance testing of VHDL designs. In M. Kim, S. Kang, and K. Hong, editors, Testing of Communicating Systems, volume 10, pp.432-447. Chapman and Hall, 1997.

[19] D. Sarnoff Research Center. Sarnoff Compliance Bitstreams for MPEG2 Video. Version 1.1, user's manual, 1996.

[20] D.P. Sidhu, T.K. Leung. Formal methods in protocol testing, a detailed study, IEEE trans. on Softw. Eng., Vol. 15, No. 4, pp.413-426 (1989).

[21] Telelogic SDT3.1, Getting Started, Telelogic AB, nov. 1996.

[22] H.P.E. Vranken. Design for test and debug in harware/software systems, Ph.D. Thesis, Eindhoven University of Technology, ISBN 90-386-04408 (1998).

[23] S.T. Vuong, W.Y.L. Chan, M.R. Ito. The UIOv-Method for Protocol Test Sequence Generation, Proceedings 2nd Internal Workshop on Protocol Test Systems, pp.161-176, North-Holland, 1990. 